\title{
Time Response of Shape Memory Alloy Actuators
}

\author{
PAVEl L. PotaPoV* AND EdSON P. DA SiLVA \\ Technische Universität Berlin, Institute für Verfahrenstechnik, Salzufer 17/19, D-10587, Berlin, Germany
}

\begin{abstract}
Force/displacement actuators with the high output power and time response can be fabricated from shape memory wires or ribbons. Typically Ni-Ti shape memory alloys are used as an active material in such actuators. They are driven by Joule heating and air convection cooling. In the present work, the time response of various types of $\mathrm{Ni}$-Ti actuators having different transformation temperatures and geometrical sizes, is studied systematically under conditions of free and forced air convection.

The simple analytical model for calculating the time response is developed which accounts for the latent heat and thermal hysteresis of transformation. For all the types of considered actuators, the calculated time response is in a good agreement with that observed experimentally. Finally, on the base of the suggested model, we present the time response of Ni-Ti actuators calculated as a function of their transformation temperature and cross section dimensions.
\end{abstract}

\section{INTRODUCTION}

$\mathbf{L}$ INEAR actuators made from shape memory alloys (SMA) are capable to produce a large actuation force and/or displacement and can be applied, among other applications, as artificial muscles in various smart structures. This ability is associated with internal transformations observed in SMA, most commonly Ni-Ti based alloys. They undergo the diffusionless transformations from the martensitic $(M)$ to austenitic (A) phase on heating and the $A \rightarrow M$ transformation on cooling. The transformations are reversible and can be utilized to convert thermal energy directly into mechanical work. The dynamic thermomechanical response of shape memory alloys has been studied experimentally (Leo et al., 1993; Shaw and Kuriakides, 1995), however, these authors have addressed mainly the stress-induced transformations in SMA while practical SMA actuators normally employ thermally induced phase transformations. A typical method to trigger the transformations in SMA includes Joule heating for the $M \rightarrow A$ transition and air convection cooling for the $A \rightarrow M$ one. The total time response is composed of the time required for heating up and the time required for cooling down an actuator. In both the heating and cooling phases, the time response is strongly controlled by the thermal parameters of SMA and efficiency of the convection heat exchange between an actuator and surroundings. The dynamic behavior of SMA can be simulated in the frameworks of constitutive models proposed during last decades. A non-inclusive list is the theoretical work of Tanaka and Nagaki, 1982; Liang and Roger, 1990; Brinson, 1993; Likhachev, 1994; Boud and Lagoudas, 1996; Bekker and Brinson, 1997;

\footnotetext{
*Author to whom correspondence should be addressed. Presently with Antwerp Uni-
} versity-RUCA, EMAT, Croenenborgerlaan 171, 2020 Antwerp, Belgium.
Seelecke and Müller, 1998; Bo and Lagoudas, 1999. However, in these models, analytical description of the heat transfer normally neglects the temperature dependence of the SMA heat capacity (Brinson et al, 1996; Liang and Roger, 1997), while more comprehensive approaches accounting for the latent heat of transformation need in the complicated numerical algorithms (Bekker et al.,1998; Lagoudas and Bo, 1999).

This paper presents experimental results on impulsive Joule heating and air convection cooling of several Ni-Ti ribbons and wires having quite different transformation temperatures and geometrical sizes. Due to the small cross-section of the examined ribbons and wires, the heat transfer proceeds dominantly by convective convection between SMA actuators and surrounding air. The observed experimental results can be reasonably fitted by the proposed simple analytical model accounting for the latent heat and thermal hysteresis of transformation. By comparison between the time responses calculated with different latent heats, it is demonstrated that the effect of the transformation latent heat cannot be neglected in simulation of the dynamic behavior of SMA. The proposed model can predict the time response of SMA actuators as a function of their material parameters and geometrical sizes. Examples of such predictions are given in the last section.

\section{EXPERIMENTAL PROCEDURE AND RESULTS}

Various types of Ni-Ti ribbons were manufactured by AMT (Herk-de-Stad, Belgium), Raychem (Menlo Park, California, US) and A.V. Shelyakov (Moscow Engineering Physics Inst., Russia). Ni-Ti actuator wire (trade mark "Flexinol 90") was manufactured by Dynalloy Co. All materials show approximately the same specific heat capacity of about $0.45 \mathrm{~mJ} / \mathrm{kg} / \mathrm{K}$ at room temperature. The transforma- 
Table 1. Composition, cross section, transformation temperatures and latent heat of Ni-Ti actuators.

\begin{tabular}{|c|c|c|c|c|c|c|c|c|c|}
\hline \multirow[b]{2}{*}{ Actuator } & \multirow[b]{2}{*}{ Supplier } & \multirow{2}{*}{$\begin{array}{c}\text { Composition, } \\
\text { Atomic } \%\end{array}$} & \multirow{2}{*}{$\begin{array}{c}\text { Ribbon } \\
\text { Cross-Section, } \\
\text { mm }\end{array}$} & \multirow{2}{*}{$\begin{array}{l}\text { Wire Diameter, } \\
\mathrm{mm}\end{array}$} & \multicolumn{4}{|c|}{ Transformation Temperature $\left({ }^{\circ} \mathbf{C}\right)$} & \multirow{2}{*}{$\begin{array}{l}\text { Latent Heat } \\
\mathrm{mJ} / \mathbf{k g}\end{array}$} \\
\hline & & & & & $A_{s}$ & $A_{f}$ & $M_{s}$ & $M_{f}$ & \\
\hline$A$ & AMT & $45 \mathrm{Ni}-50 \mathrm{Ti}-5 \mathrm{Cu}$ & $0.7 \times 0.1$ & - & 26 & 60 & 38 & 10 & 13.0 \\
\hline $\mathrm{R}$ & Raychem & 40Ni-50Ti-10Cu & $1.4 \times 0.04$ & - & 56 & 65 & 50 & 43 & 13.5 \\
\hline $\mathrm{S}$ & Shelyakov & $25 \mathrm{Ni}-50 \mathrm{Ti}-25 \mathrm{Cu}$ & $1.8 \times 0.04$ & - & 54 & 63 & 58 & 48 & 13.4 \\
\hline $\mathrm{SH}$ & Shelyakov & $50 \mathrm{Ni}-35 \mathrm{Ti}-15 \mathrm{Hf}$ & $1.2 \times 0.06$ & - & 115 & 140 & 85 & 70 & 18.5 \\
\hline $\mathrm{F}$ & Dynalloy & $45 \mathrm{Ni}-50 \mathrm{Ti}-5 \mathrm{Cu}$ & - & 0.381 & 59 & 74 & 44 & 26 & 14.7 \\
\hline
\end{tabular}

tion temperatures and latent heat determined by DSC are listed in Table 1 where actuators are referred to as A, R, S, SH and $\mathrm{F}$ types depending on their manufacturer. $\mathrm{SH}$ ribbons produced by Shelyakov contained 15 at.\% of Hf in order to increase the transformation temperature. Here, the transformation start and finish temperatures are referred to as $A_{s}$ and $A_{f}$ for the $M \rightarrow A$ transformation and $M_{s}$ and $M_{f}$ for the $A \rightarrow M$ one.

$\mathrm{Ni}$-Ti actuators provide a displacement or induce a force when heated up in constant-load or constant-deflection conditions respectively. Thus, the Flexinol 90 wire of $50-60 \mathrm{~mm}$ in length was loaded by the constant weight while a differential transformer measured the deflection of the wire on heating and cooling. Since only short Ni-Ti ribbons (10-20 mm) with uniform cross-section were available, the constant-load mode did not provide the sufficient deflection. Thus, the constant-deflection mode with measuring the induced load was selected in the case of ribbons. To induce the shape memory effect the ribbons were pre-deformed at room temperature and then placed between two motionless grips with the inter-grip distance of $12 \mathrm{~mm}$. One of the grips was connected with a load cell sensor allowing us to control the load induced by the ribbon. For each actuator, two series of experiments were executed: quasi-static and dynamic heating/cooling.

In quasi-static experiments, the Ni-Ti ribbons were slowly heated/cooled with the precise control of the temperature over its length. On heating from $A_{s}$ to $A_{f}$ points, the $M \rightarrow A$ transformation occurs and induces the load between the grips. On cooling from $M_{s}$ to $M_{f}$, the ribbons undergo the $A \rightarrow$ $M$ transition resulting in a reset of the induced load. The maximal load slightly changes with the heating/cooling cycling and it is stabilized in 5-10 cycles. Figure 1 shows the load-temperature curves of $\mathrm{A}, \mathrm{R}, \mathrm{S}$ and $\mathrm{SH}$ ribbons obtained after 10 complete cycles. The shape of the transformation loop is similar in all the examined ribbons although the transformation points and hysteresis vary significantly due to different composition and treatment of the ribbons.

Slow heating of the Flexinol 90 wires results in their deflection, which is reset on forthcoming cooling. Two cycles of heating-cooling are sufficient to stabilize the deflection-temperature curves of the wires. Figure 2(a) shows the stabilized curves under the different loads. The deflection in the austenitic state is set as zero because it corresponds to the "memorized shape" of the wire and does not depend on the load applied. As demonstrated in Figure 2(b), the transformation points of the wire in constant-load conditions increase with increasing applied load.

The variation of the wire deflection on cooling/heating is proportional to the amount of martensite in a sample. For constant-load conditions, Liang and Roger, 1990 suggested the cosine empirical dependence of the martensite fraction $n$ on temperature. Assuming the $M_{s}$ and $M_{f}$ temperature to be

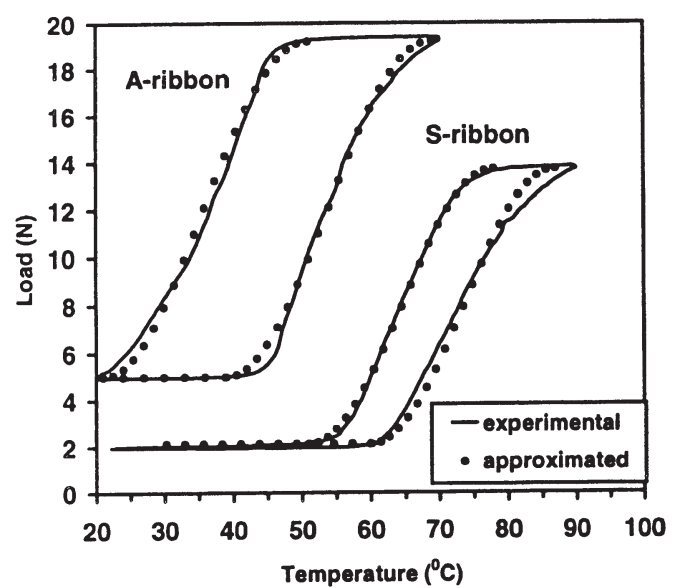

(a)

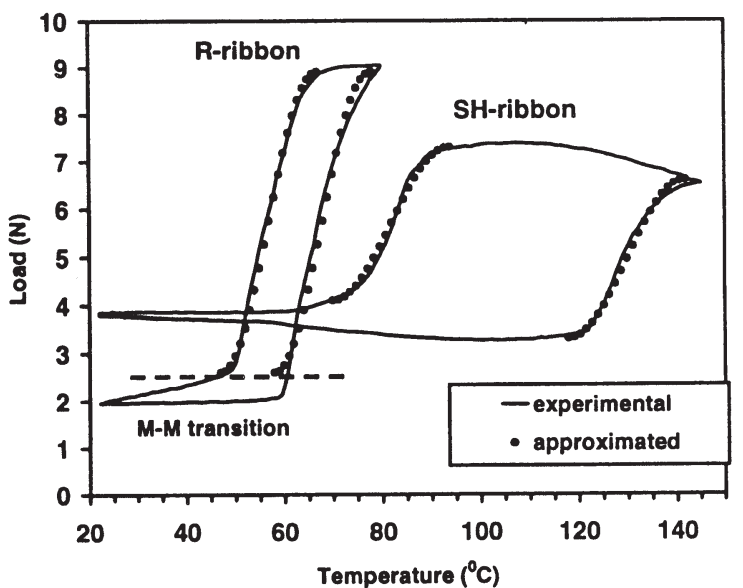

(b)

Figure 1. Load-temperature curves of $A, S$ (a) and $R, S H$ (b) ribbons obtained at heating/cooling rate $5^{\circ} \mathrm{C} / \mathrm{min}$ in constant-deflection conditions. Experimental curves are fitted by Equation (1) assuming that the transformation-induced load is proportional to the amount of martensite in the ribbon. 


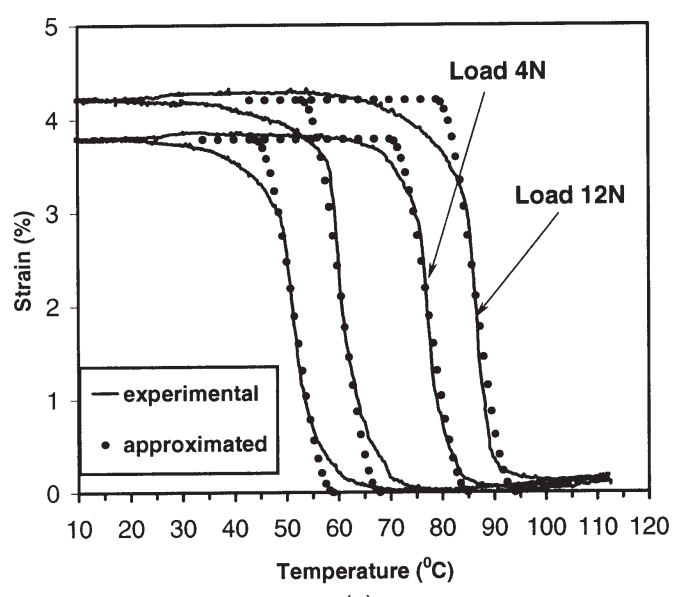

(a)

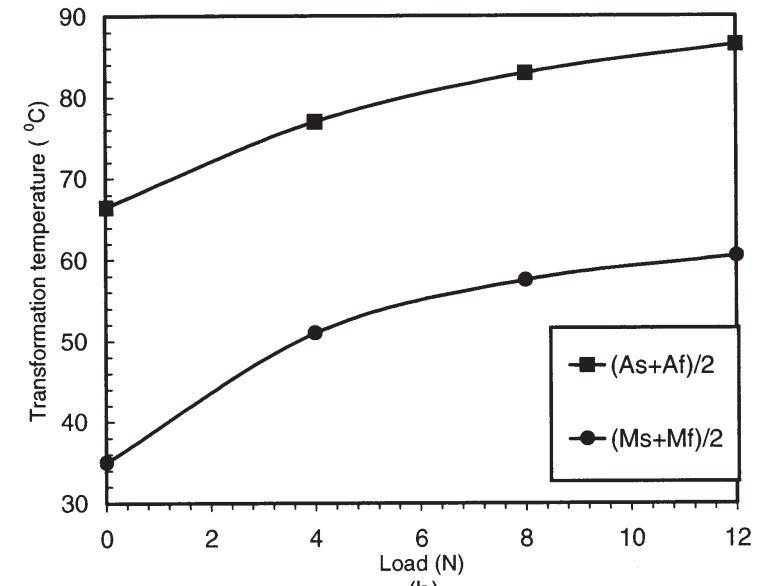

(b)

Figure 2. (a) Deflection-temperature curves of $F$ wire obtained at heating/cooling rate $5^{\circ} \mathrm{C} / \mathrm{min}$ in constant-load conditions. Experimental curves are fitted by Equation (1) assuming that the transformation-induced strain is proportional to the amount of martensite in the wire and (b) change of the transformation temperatures in F wire with the applied load.

load-dependent, the Roger-Liang kinetic law for cooling can be written as

$$
\begin{gathered}
n=0 \quad \text { at } T<M_{s} \\
n(T)=\frac{1}{2}\left[\cos \left(\pi \frac{T-M_{f}}{M_{s}-M_{f}}\right)+1\right] \quad \text { at } M_{f}<T<M_{s} \\
n=1 \quad \text { at } T<M_{f}
\end{gathered}
$$

The analogous empirical dependence can be built for heating with replacing the $M_{s}$ and $M_{f}$ temperatures for the $A_{f}$ and $A_{s}$ temperatures correspondingly. The empirical deflection-temperature curves for Flexinol wire are plotted by dots in Figure 2(a). Fitting the experimental curves by Equation (1) shows the reasonable match at $n<0.8-0.9$. At higher $n$, the experimental curves deviate from the empirical ones, which might be associated with the complex internal stresses typical for Flexinol wire. In the case of Ni-Ti ribbons, the coupling between the induced load and the martensite fraction results in an increase of transformation temperatures, especially for $A_{f}$ and $M_{s}$ points. Indeed, the comparison of the curves in Figure 1 with the data in Table 1 reveals an increase of all the transformation points and the transformation ranges $\left(A_{f}-A_{s}\right)$ and $\left(M_{s}-M_{f}\right)$. However, Figure 1 demonstrates that the empirical dependence (1) with the modified $M_{s}, M_{f}, A_{f}$ and $A_{s}$ parameters can be still used to build the load-temperature curves in constant-deflection conditions. In this case, $M_{s}, M_{f}, A_{f}$ and $A_{s}$ temperatures should be calculated from the well-known Clausius-Clapeyron equation or taken from experiments. Out of the transformation range, some ribbons show the small negative slope of the load-temperature curves due to thermal expansion. The $\mathrm{R}$ ribbons indicate the more complex behavior with a positive slope of the load below $M_{f}$ temperature. Moreover, a positive slope is observed even on cooling far below room tempera- ture. That is a result of the transition between different types of martensite reported in a NiTi-10\% Cu alloy, which was the material of the $\mathrm{R}$ ribbons. In this paper, we neglect the martensite $\rightarrow$ martensite transition because it is associated with the much smaller latent heat and induced load than the $A \rightarrow M$ one.

In dynamic experiments with $\mathrm{Ni}$-Ti ribbons, heating was executed by sending the short rectangular DC impulse through the actuators followed by cooling with free air convection. The load-time protocol was continuously recorded during heating/cooling whilst the temperature of the ribbon was not controlled. Assuming that the load-temperature curves do not change significantly with a heating/cooling rate, the temporal evolution of temperature can be extracted from the load-time protocol and the quasi-static data in Figure 1. Figure 3 shows the results of dynamic experiments when the duration of the heating impulse was varied from 0.1 to $6 \mathrm{~s}$. The duration of impulse is referred as the heating time $t_{H}$. At each impulse duration, the value of DC was precisely adjusted in order to reach the same maximal temperature as that for the quasi-static experiments $\left(70^{\circ} \mathrm{C}\right.$ in the $\mathrm{A}, 80^{\circ} \mathrm{C}$ in the $\mathrm{R}, 90^{\circ} \mathrm{C}$ in the $\mathrm{S}$ and $145^{\circ} \mathrm{C}$ in the $\mathrm{SH}$ ribbons). For instance, $\mathrm{R}$ ribbons were heated up to $80^{\circ} \mathrm{C}$ for $6 \mathrm{~s}$ by sending $4.008 \mathrm{~A}$ and for $0.1 \mathrm{~s}$ by sending $7.330 \mathrm{~A}$. The load corresponding to the maximal temperature is marked as the set point. The other marker indicates the level when $90 \%$ of the transformation-induced load is reset by cooling. Note that in the case of cooling the SH ribbons [Figure 1(b)], the load slightly increases in the range of $90-145^{\circ} \mathrm{C}$ due to thermal contraction and then decreases in the $70-90^{\circ} \mathrm{C}$ range due to the $\mathrm{A} \rightarrow \mathrm{M}$ transformation. As seen in Figure 3(d), this feature is reproduced in dynamic experiments.

In dynamic experiments with Flexinol 90 wire, the deflection-time protocol was recorded as shown in Figure 4 and processed using the quasi-static curves in Figure 2. Again, the DC value was precisely adjusted in order to reach the deflection value corresponding to $A_{s}$ temperature. As the wire 


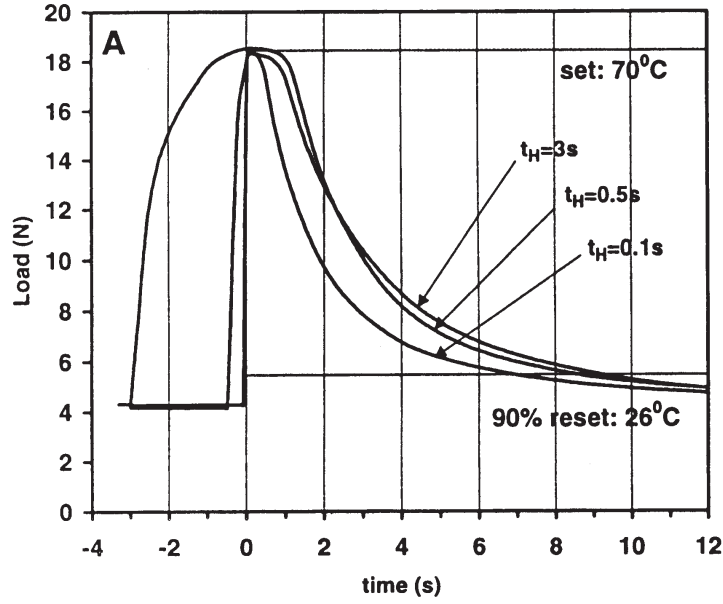

(a)

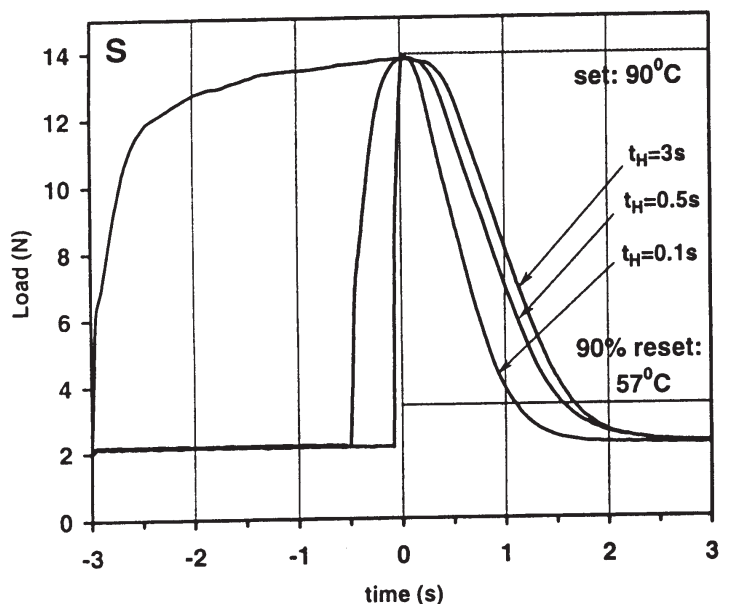

(c)

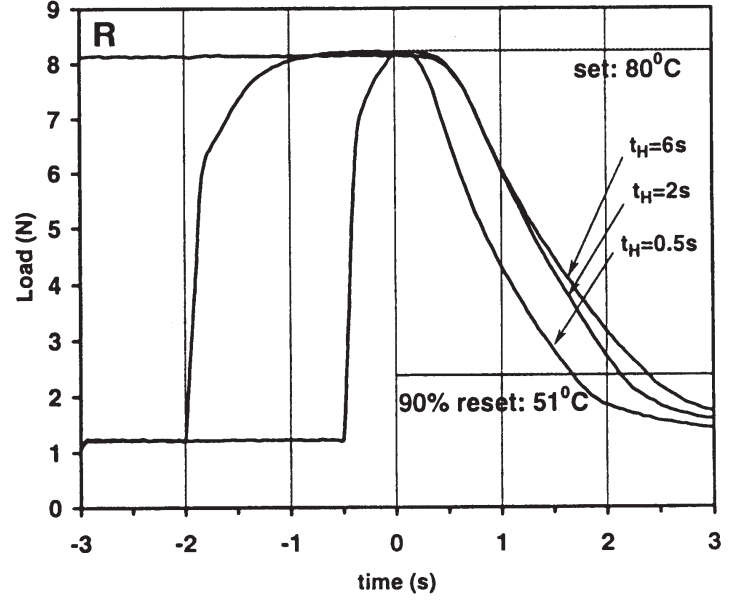

(b)

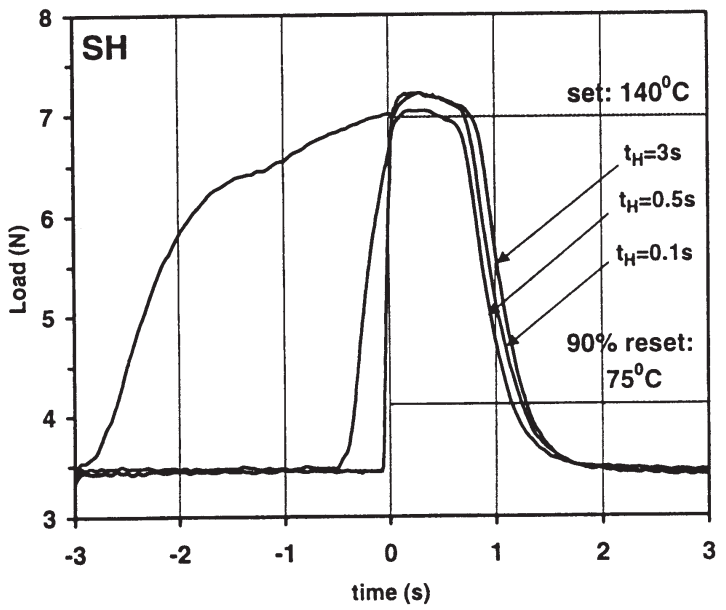

(d)

Figure 3. Impulsive Joule heating of $A(a), R(b), S(c)$ and $S H$ (d) ribbons followed by free air convention cooling. The duration of impulse is denoted by $\mathrm{t}_{\mathrm{H}}$. For convenience, each curve is shifted along the time-axis such as cooling starts always at $\mathrm{t}=0$ regardless of the heating duration.

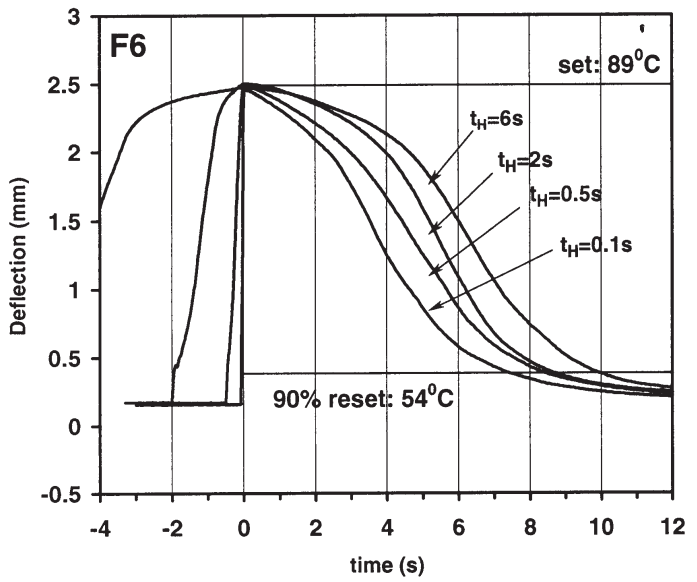

(a)

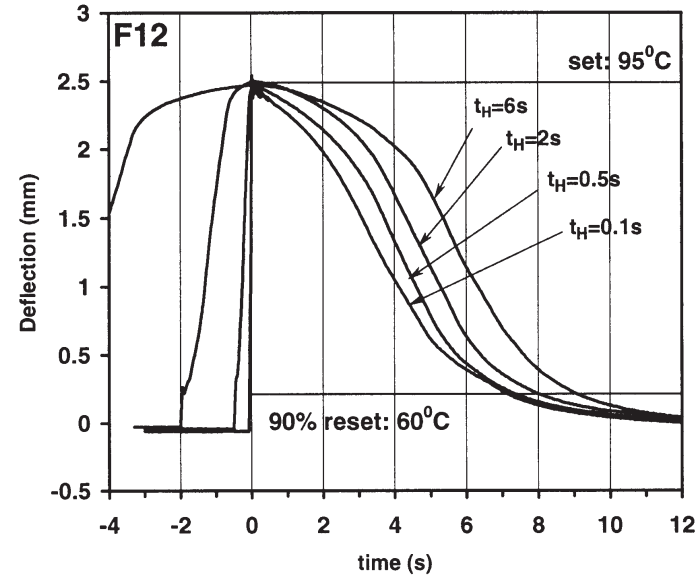

(b)

Figure 4. Impulsive Joule heating of $F$ wire under the load of $6 \mathrm{~N}(\mathrm{a})$ and $12 \mathrm{~N}$ (b) followed by free air convection cooling. The duration of impulse is denoted by $\mathrm{t}_{\mathrm{H}}$. For convenience, each curve is shifted along the time-axis such as cooling starts always at $\mathrm{t}=0$ regardless of the heating duration. 
deflection changes slightly above $A_{s}$, the special attention was paid to avoid overheating of wire. The DC value was increased by small steps with recording the temperature achieved. The final DC value was set by extrapolating the temperature to the $A_{f}$ point.

We refer the time required for cooling from the set point to the $90 \%$ reset point as the cooling time $t_{C}$. The cooling time varies from the actuator to the actuator being the maximal in $\mathrm{F}$ wire under the load of $6 \mathrm{~N}$ and the minimal in $\mathrm{SH}$ ribbons. Also the cooling time decreases slightly with decreasing the duration of DC impulse.

The cooling process can be forced by flowing air from an external fan. Figures 5 and 6 show the examples of the experiments when the actuators were exposed to a permanent airflow with the rate of $2.5 \mathrm{~m} / \mathrm{s}$. Comparing with free convection, the cooling time decreases by a factor 3 in ribbons and by a factor 5 in wires. At the same time, the higher thermal losses result in 2-3 times higher power consumption during the heating phase as compared with actuation when the fan is off.

\section{MODELING}

The existing models can successfully simulate the dynamic behavior of SMA using numerical algorithms (Bekker et al, 1998; Lagoudas and Bo, 1999). In contrast, in this section, we will develop the simple model suitable for the easy analytical prediction of the time response of SMA actuators. Similar analytical modeling has been suggested by Brailovski et al., 1996. They, however, employed the polynomial kinetic law, which needs a number of experimentally determined coefficients. In this paper, for the cooling process, we will utilize the Liang-Roger kinetic law (1) that depends on only the parameters $M_{s}$ and $M_{f}$. The same equation can be applied for the heating process with replacing the $M_{s}$ and $M_{f}$ temperatures for the $A_{f}=M_{s}+\Delta T$ and $A_{s}=M_{f}+\Delta T$ temperatures correspondingly, where $\Delta T$ is the thermal hys-

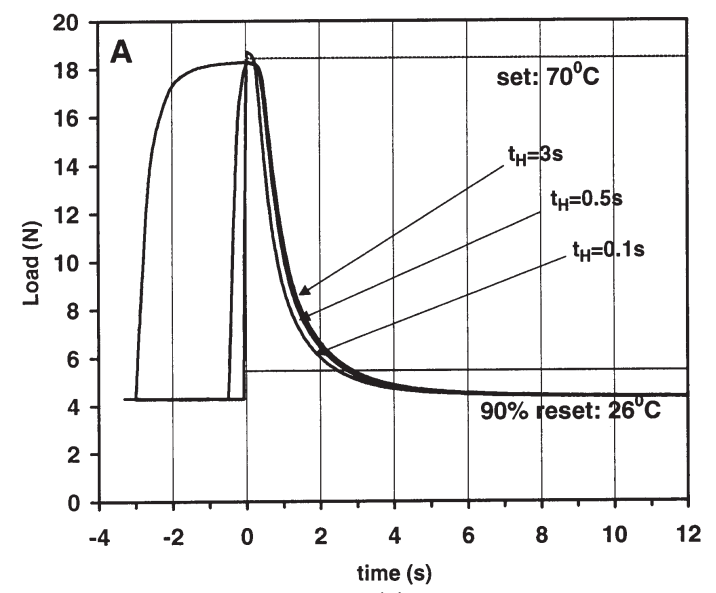

(a) teresis of transformation. Although during actuation the heating phase precedes the cooling one, we shall consider firstly the cooling phase, which is easier to model.

\section{Cooling Phase}

During the cooling phase, a Ni-Ti actuator should be cooled down from the $A_{f}$ temperature. If we assume that a single temperature characterizes the temperature of an actuator and neglect heat conduction at the actuator ends, the heat transfer problem is defined by

$$
-\rho V\left(c_{p} d T+\Delta H d n\right)=h F\left(T-T_{0}\right) d t \quad \text { at } \quad M_{s}<T<A_{f}
$$

where $\rho$ is the density of $\mathrm{Ni}-\mathrm{Ti}$ (about $6500 \mathrm{~kg} / \mathrm{m}^{3}$ ), $c_{p}$ is the $\mathrm{Ni}-\mathrm{Ti}$ specific heat capacity in the absence of transformations, $V$ and $F$ are the volume and the free surface of an actuator respectively, $\Delta H$ is the integral latent heat for transformation on cooling, $h$ is the coefficient of the heat exchange between an actuator and surroundings, $T$ is the temperature of a Ni-Ti actuator and $T_{0}$ is the temperature of surroundings. Introducing, after Wirtz et al., 1995, dimensionless variables:

dimensionless time

$$
T^{\prime}=\frac{T-M_{f}}{M_{s}-M_{f}} \quad t^{\prime}=\frac{h F}{c_{p} \rho V} t
$$

dimensionless difference between the transformation and room temperatures

$$
S=\frac{M_{f}-T_{0}}{M_{s}-M_{f}}
$$

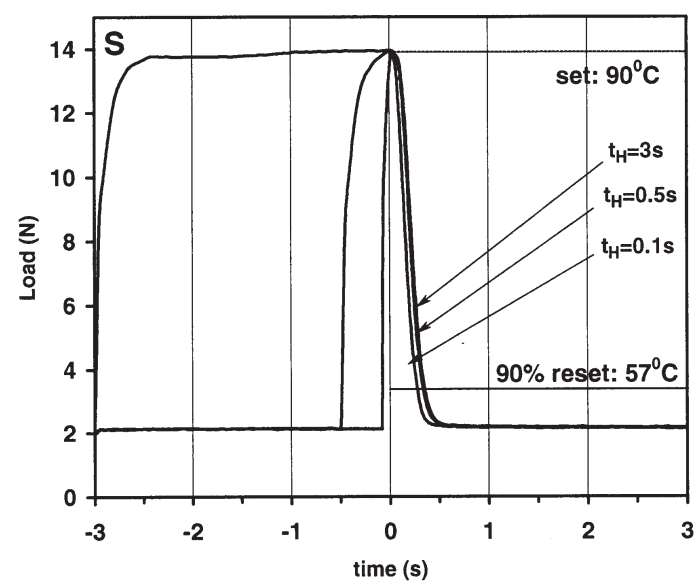

(b)

Figure 5. Impulsive Joule heating of $A(a)$ and $S$ (b) ribbons followed by forced air convection cooling with a flow rate of $2.5 \mathrm{~m} / \mathrm{s}$. The duration of impulse is denoted by $\mathrm{t}_{\mathrm{H}}$. For convenience, each curve is shifted along the time-axis such as cooling starts always at $\mathrm{t}=0$ regardless of the heating duration. 


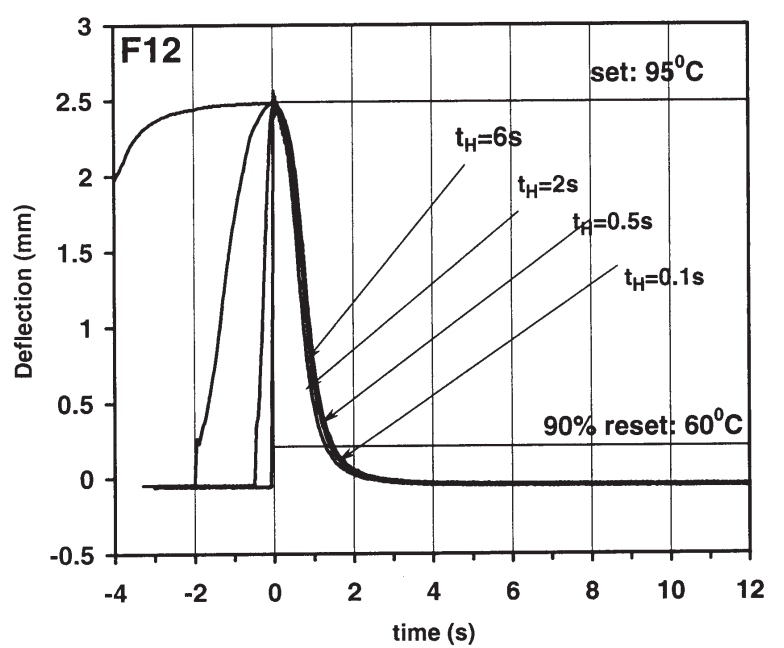

Figure 6. Impulsive Joule heating of $F$ wire under the load of $12 \mathrm{~N}$ followed by forced air convection cooling with a flow rate of $2.5 \mathrm{~m} / \mathrm{s}$. The duration of impulse is denoted by $\mathrm{t}_{\mathrm{H}}$. For convenience, each curve is shifted along the time-axis such as cooling starts always at $\mathrm{t}=0$ regardless of the heating duration.

dimensionless transformation latent heat

$$
H=\frac{\Delta H}{c_{p} \rho V\left(M_{s}-M_{f}\right)}
$$

and dimensionless transformation hysteresis

$$
G=\frac{\Delta T}{M_{s}-M_{f}}=\frac{A_{f}-M_{s}}{M_{s}-M_{f}}
$$

and assuming that the martensite volume fraction depends on temperature as (1), the Equation (2) is rewritten as

$$
\begin{aligned}
& -\frac{d T^{\prime}}{d t^{\prime}}=T^{\prime}+S \text { at } \quad 1<T^{\prime}<1+G \text { and at } T^{\prime}<0 \\
& -\left(1+\frac{\pi}{2} H \sin \left(\pi T^{\prime}\right)\right) \frac{d T^{\prime}}{d t^{\prime}}=T^{\prime}+S \text { at } 0<T^{\prime}<1
\end{aligned}
$$

Integrating Equation (3) with the boundary condition $T^{\prime}=$ $1+G$ at $t^{\prime}=0$ we obtain the simplest temperature-time curve:

$$
t^{\prime}=-\ln \left(T^{\prime}+S\right)+\ln (1+G+S) \text { at } 1<T^{\prime}<1+G
$$

In the regime $0<T^{\prime}<1$, integration of Equation (4) with the boundary condition $T^{\prime}=1$ at $t^{\prime}=t_{1}^{\prime}=-\ln (1+S)+\ln (1+G$ $+S$ ) gives the solution

$$
\begin{gathered}
t^{\prime}=t_{1}^{\prime}-\ln \left(T^{\prime}+S\right)+\ln (1+S)-\frac{\pi}{2} H\left\{\operatorname { c o s } ( \pi S ) \left[\operatorname{Si}\left(\pi\left(T^{\prime}+S\right)\right)\right.\right. \\
\left.-\operatorname{Si}(\pi(1+S)]-\sin (\pi S)\left[C i\left(\pi\left(T^{\prime}+S\right)\right)-C i(\pi(1+S))\right]\right\} \\
\text { at } \quad 0<T^{\prime}<1
\end{gathered}
$$

where

$$
\operatorname{Si}(x)=\int_{0}^{x} \frac{\sin (y)}{y} d y \quad \text { and } \quad \operatorname{Ci}(x)=\int_{0}^{x} \frac{\cos (y)}{d} d y
$$

are the sine and cosine integral functions incorporated as standard in most mathematical software. The formula (6) is valid at any $S$ parameter. We note that at $S>1$, the heat transfer problem can be solved even without using the special functions. Dividing both the sides of (4) by $\left(S+T^{\top}\right)$ and approximating the $1 /\left(S+T^{\prime}\right)$ term as a linear function of temperature in the vicinity of the point $T^{\prime}=0.5$, Equation (4) can be rewritten as

$$
-\left(\frac{1}{T^{\prime}+S}+\frac{\pi}{2} H \frac{1+S-T^{\prime}}{(0.5+S)^{2}} \sin \left(\pi T^{\prime}\right)\right) \frac{d T^{\prime}}{d t^{\prime}}=1 \quad \text { at } \quad 0<T^{\prime}<1
$$

Linearisation of the $1 /\left(S+T^{\prime}\right)$ term is exact only in the small vicinity of the point $T^{\prime}=0.5$. However, the deviations of Equation (7) from Equation (4) at $T^{\prime}=0$ and $T^{\prime}=1$ are still negligible because of $\sin \left(\pi T^{\prime}\right)$ goes to 0 at these points. Integrating Equation (7) with the boundary condition $T^{\prime}=1$ at $t^{\prime}=t_{1}^{\prime}$ we obtain the simplified solution

$$
\begin{gathered}
t^{\prime}=t_{1}^{\prime}-\ln \left(T^{\prime}+S\right)+\ln (1+S) \\
+\frac{H}{2(0.5+S)^{2}}\left[\left(1+S-T^{\prime}\right) \cos (\pi T)^{\prime}+\frac{1}{\pi} \sin \left(\pi T^{\prime}\right)+S\right] \\
\text { at } 0<T^{\prime}<1
\end{gathered}
$$

Figure 7 shows the calculated dimensionless cooling curves with the various $H$ and $S$ parameters. Evidently the lower $S$ results in the slower cooling process. As seen from Figure 7(b), the $H$ parameter, which is a dimensionless measure of the transformation latent heat, also strongly affects the cooling rate. The typical for Ni-Ti $H$ values of 1-2.5 results in a 2-3 times increase of the cooling time comparing with the calculation not accounting for the latent heat. Note that the curves calculated with formula (8) show the only minor deviation from the exact solution (6).

At $t_{0}^{\prime}=t_{1}^{\prime}-\ln S+\ln (1+S)+H /(0.5+S)$, the transformation finishes and the heat transfer is again defined by Equation (3). Finding the solution in the regime $T^{\prime}<0$ is evident.

\section{Heating Phase}

During the heating phase, DC running through an actuator induces heating. When the constant electrical power $W$ is supplied to an actuator, the heat transfer problem is defined by

$$
-\frac{d T^{\prime}}{d t^{\prime}}=T^{\prime}+S-P \quad \text { at } \quad T^{\prime}<G
$$


$\mathrm{H}=1.5$

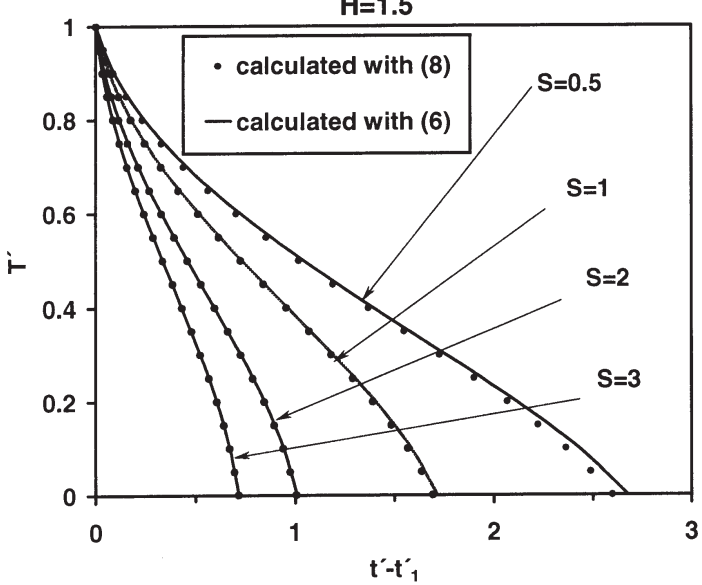

(a)

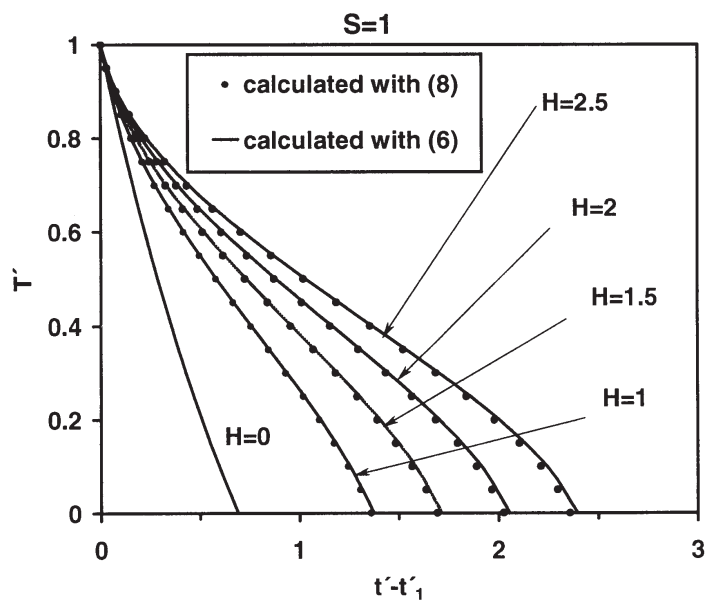

(b)

Figure 7. Calculated cooling profiles in dimensionless terms (see definition in text).

$$
\begin{gathered}
-\left(1+\frac{\pi}{2} H \sin \left(\pi\left(T^{\prime}-G\right)\right)\right) \frac{d T^{\prime}}{d t^{\prime}}=T^{\prime}+S-P \\
\text { at } G<T^{\prime}<1+G
\end{gathered}
$$

where $P$ is the dimensionless power $P=W / h F\left(M_{s}-M_{f}\right)$. Taking the boundary condition as $T^{\prime}=1+G$ at $t^{\prime}=0$ we obtain the following solution:

$$
\begin{gathered}
t^{\prime}=-\ln \left(P-S-T^{\prime}\right)+\ln (P-S-1-G) \\
+\frac{H}{2(0.5+S+G-P)^{2}}\left[\left(1+S+2 G-P-T^{\prime}\right) \cos \left(\pi\left(T^{\prime}-G\right)\right)\right. \\
\left.+\frac{1}{\pi} \sin \left(\pi\left(T^{\prime}-G\right)\right)+S+G-P\right] \text { at } G<T^{\prime}<1+G
\end{gathered}
$$

In the regime $T^{\prime}<G$, integration of Equation (9) with the appropriated boundary conditions gives again the logarithmic curve, which is smoothly connected with Equation (11) at $T^{\prime}=G$.

\section{Comparison with Experiment}

The accurate comparison with experiment depends on the heat exchange coefficient $h$ between a Ni-Ti actuator and surroundings. This coefficient can be calculated from the standard theory of the convective heat exchange with accounting geometrical configuration of an actuator. In the present work, we used the formulas recommended by Beitz and Küttner, 1995 for plain ribbons and cylindrical wires subjected by air free or forced convection. The thermal constants of air were taken from Baehr and Stephan, 1996. The calculated results are listed in Table 2 in comparison with the experimental cooling time. Despite of the rough assumptions made, the calculations reproduce reasonably the experimental data for all examined actuators and cooling methods. Figure 8 compares the calculated and experimental temperature-time profiles on cooling. Both the calculations and experiments show the pronounced delay on the cooling

\begin{tabular}{|c|c|c|c|c|c|c|c|c|c|c|c|}
\hline \multirow[b]{3}{*}{ Actuator } & \multirow[b]{3}{*}{$M_{s},{ }^{\circ} \mathbf{C}$} & \multirow[b]{3}{*}{$M_{f},{ }^{\circ} \mathrm{C}$} & \multirow[b]{3}{*}{$T_{0},{ }^{\circ} \mathrm{C}$} & \multirow[b]{3}{*}{$\Delta \boldsymbol{T},{ }^{\circ} \mathbf{C}$} & \multicolumn{5}{|c|}{ Calculated } & \multicolumn{2}{|c|}{ Experimental } \\
\hline & & & & & \multirow[b]{2}{*}{$t_{C}^{\prime}$} & \multicolumn{2}{|c|}{ Free Convection } & \multicolumn{2}{|c|}{ Forced Convection } & \multirow{2}{*}{$\begin{array}{c}\begin{array}{c}\text { Free } \\
\text { Convection }\end{array} \\
t_{c}, \text { sec. }\end{array}$} & \multirow{2}{*}{$\begin{array}{c}\begin{array}{c}\text { Forced } \\
\text { Convection }\end{array} \\
t_{C}, \text { sec. }\end{array}$} \\
\hline & & & & & & $h, \mathrm{~W} / \mathrm{m}^{2} / \mathrm{K}$ & $t_{c}$, sec. & $h, \mathrm{~W} / \mathrm{m}^{2} / \mathrm{K}$ & $t_{c}$, sec. & & \\
\hline$A^{a}$ & 51 & 21 & 20 & 18 & 4.27 & 67.2 & 9.3 & 231 & 2.7 & $8.5^{c}$ & $2.9^{d}$ \\
\hline$R^{b}$ & 67 & 47 & 22 & 11 & 1.59 & 42.2 & 2.0 & 148 & 0.6 & $2.1^{c}$ & $0.5^{\mathrm{e}}$ \\
\hline$S^{b}$ & 78 & 51 & 22.5 & 9 & 1.45 & 45.6 & 2.0 & 163 & 0.6 & $1.7^{\mathrm{d}}$ & $0.4^{d}$ \\
\hline $\mathrm{SH}^{\mathrm{b}}$ & 94 & 70 & 22 & 48 & 1.27 & 56.1 & 1.6 & 177 & 0.5 & $1.3^{\mathrm{c}}$ & $0.4^{d}$ \\
\hline $\mathrm{F} 6^{\mathrm{b}}$ & 62 & 47 & 20 & 26 & 1.74 & 52.8 & 9.2 & 249 & 1.9 & $9.9^{c}$ & $1.7^{\mathrm{c}}$ \\
\hline $\mathrm{F} 12^{\mathrm{b}}$ & 68 & 53 & 20 & 26 & 1.50 & 52.8 & 7.9 & 249 & 1.7 & $8.0^{c}$ & $1.5^{\mathrm{c}}$ \\
\hline
\end{tabular}
curves due to the released transformation latent heat.

The suggested model, however, does not predict any de-

Table 2. Calculated dimensionless cooling time $t_{c}^{\prime}$, heat exchange coefficient $(\mathrm{h})$, and dimensional cooling time $\left(\mathrm{t}_{\mathrm{c}}\right)$ in comparison with the experimental cooling time.

${ }^{\mathrm{C} C a l c u l a t e d}$ with Equation (6)

${ }^{\mathrm{b}}$ Calculated with Equation (8)

cHeating impulse $2 \mathrm{~s}$.

dHeating impulse $3 \mathrm{~s}$.

eHeating impulse $6 \mathrm{~s}$. 


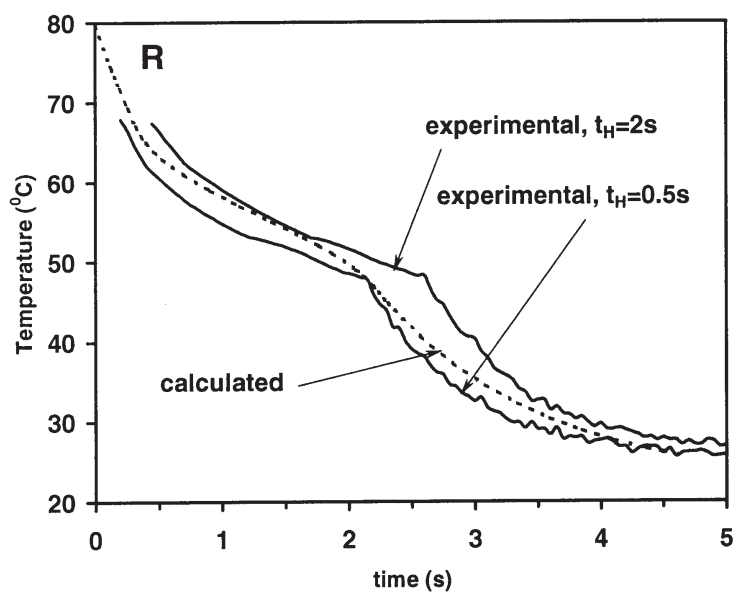

(a)

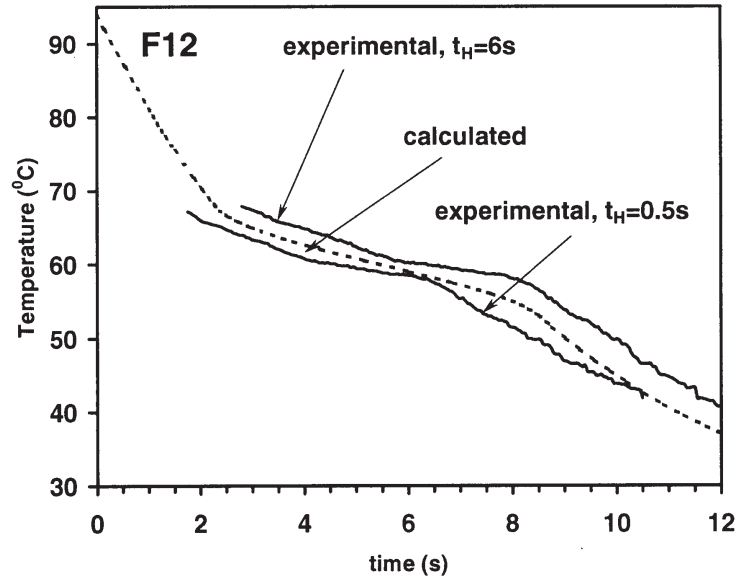

(b)

Figure 8. Comparison of the experimental cooling profiles with the calculated ones for (a) $R$ ribbon and (b) $F$ wire under the load of $12 \mathrm{~N}$.

pendence of the cooling time on the heating impulse duration. That is because the model assumes $h$ to be constant and equal to the steady value defined by the stationary convective heat exchange. In reality, as demonstrated by Polidori et al., 1998, the heat exchange between impulsively heated samples and surroundings can show unsteady behavior. At the beginning of heating, the heat exchange coefficient is very high and then it decreases rapidly to reach the steady value. The duration of the transient regime depends on geometry of a sample and properties of surrounding fluid. For actuators examined in the present work, the transient regime takes approximately $2 \mathrm{~s}$. That can be concluded from the analysis of the heating branches of the load (deflection) curves in Figures 3 and 4 . The load (deflection) reaches the steady level in approximately $2 \mathrm{~s}$ after switching DC on. In the case of the 0.1 and $0.5 \mathrm{~s}$ impulses, the current is cut before the stationary conditions are reached, thus, further cooling proceeds under conditions of the unsteady heat exchange with the increased heat exchange coefficient. Figure 9 demonstrates the effect of the heating impulse duration on the forthcoming cooling

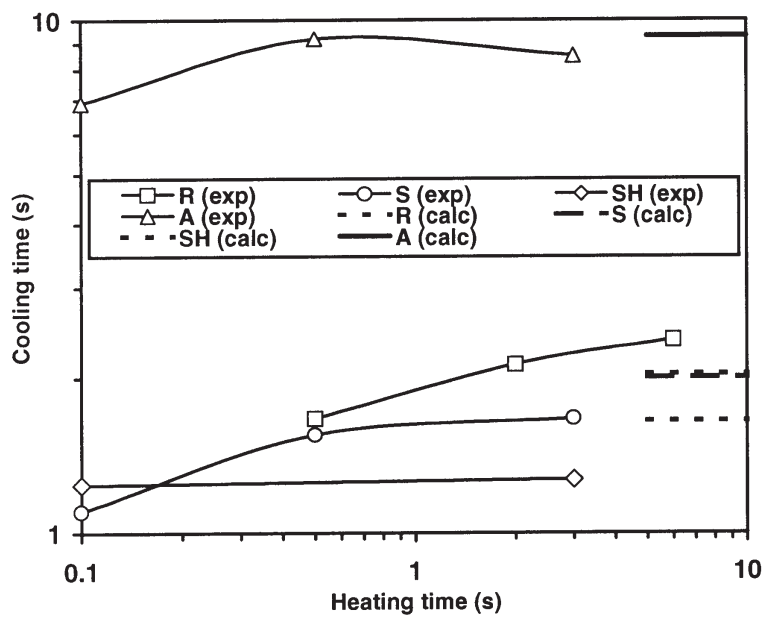

Figure 9. Effect of the heating impulse duration on the forthcoming cooling process in the case of free air convection. process. The cooling time firstly increases with the heating time and then comes to saturation at $t_{H}=2-6 \mathrm{~s}$. These saturation values can be adequately modeled in terms of stationary heat exchange as demonstrated above. The modeling of the shorter heating impulses $(0.1-0.5 \mathrm{~s})$ should however address the unsteady heat transfer problem, which can not be solved analytically.

\section{Effects of Transformation Temperature, Geometrical Size and Heating Current on Time Response}

Figure 10 shows the calculated heating time of Flexinol 90 wire as a function of the applied current. An increase of DC by factor 3 results in an order of magnitude decrease of the heating time. Thus, the time response on heating can be as small as desired when the powerful heating impulse is applied. That agrees with the experimental observations. For instance, Shelyakov et al.,1994 has demonstrated that the transformation can be initiated in thin $\mathrm{Ni}$-Ti ribbons heated up electrically for the time as small as $0.005 \mathrm{~s}$.

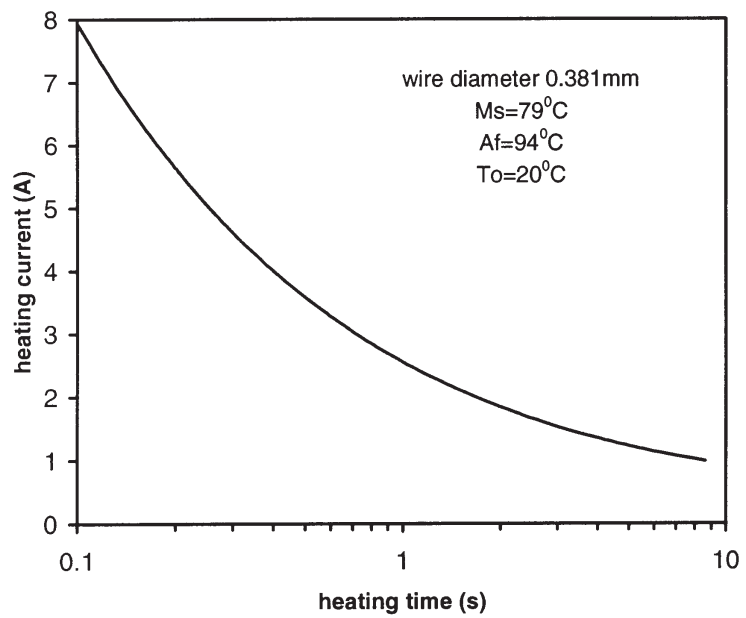

Figure 10. The calculated heating time versus heating $D C$ value for $F$ wire under the load of $12 \mathrm{~N}$. 


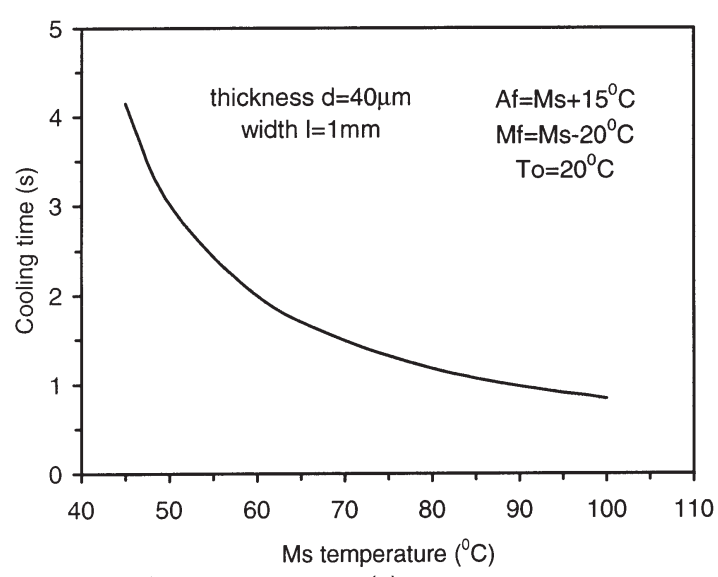

(a)

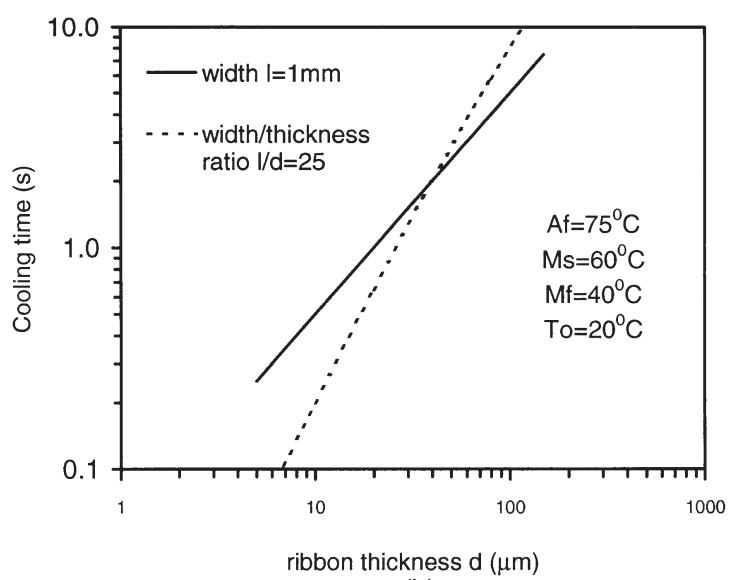

(b)

Figure 11. Predicted cooling time for a Ni-Ti actuator as a function of $\mathrm{M}_{\mathrm{s}}$ temperature (a) and thickness (b). The transformation latent heat is always assumed to be $14 \mathrm{~J} / \mathrm{g}$.

As the heating time can be extremely short at the sufficient electrical power applied, the total time response of Ni-Ti actuators is controlled mainly by the cooling time. The Equations (5, 6, and 8) enable us to estimate the cooling time of Ni-Ti actuators as a function of their geometrical size and transformation temperature. Figure 11(a) shows the effect of the $M_{s}$ temperature on the cooling time in Ni-Ti ribbons. To provide rapid actuation at room temperature, the $M_{s}$ should stay in the range of $60-80^{\circ} \mathrm{C}$ while an increase of $M_{s}$ above $80^{\circ} \mathrm{C}$ results in an only small further improvement of the time response. This conclusion is consistent with the experimental data by Yaeger, 1990 who studied the time response when the surrounding temperature was varied whilst the transformation temperature was fixed.

A decrease of the actuator cross section demonstrates a more dramatic effect on the time response. Figure 11(b) shows the cooling time as a function of a ribbon thickness (note the logarithmic scale). We conclude that ribbons with a thickness of few microns can provide the time response of about $0.1 \mathrm{~s}$. That agrees with the experimental data observed

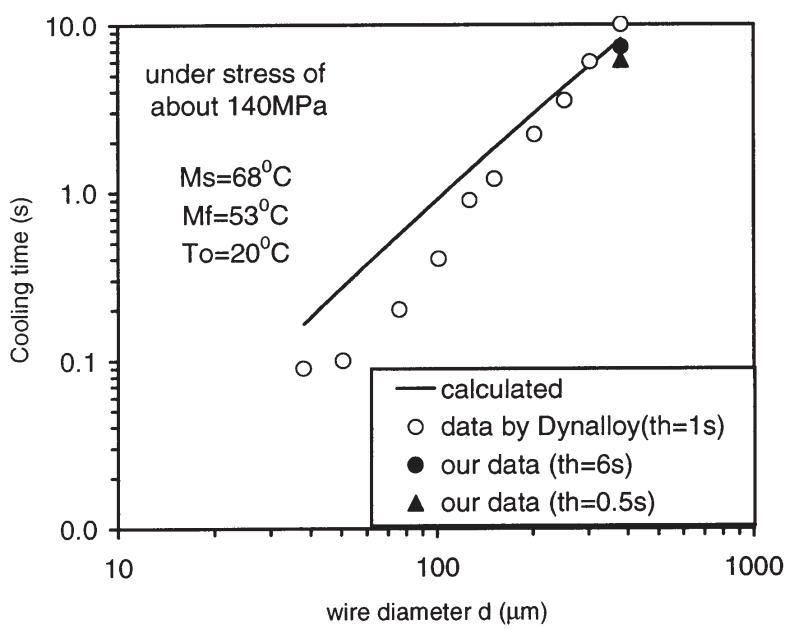

Figure 12. Predicted and experimental cooling time for Flexinol 90 wire as a function of wire diameter. The transformation latent heat is assumed to be $14.7 \mathrm{~J} / \mathrm{g}$. on sputter deposited Ni-Ti films (Miyazaki,1999). In the case of wire, the cooling time also decreases rapidly with decreasing wire diameter. Figure 12 displays good agreement between the calculated time response vs. wire diameter and technical information supplied by Dynalloy Co.

So, the simple analytical model can reasonably simulate the heating and cooling processes in shape memory actuators. This model operates with simple quantities easy extracted from experiments or manufacturer technical data.

\section{CONCLUSIONS}

1. The heating and cooling processes in shape memory actuators can be reasonably simulated by the simple analytical model proposed.

2. The actuation frequency of shape memory actuators is controlled mainly by the cooling time whilst the heating time can be reduced unlimitedly by increasing the supplied power.

3. The actuation frequency of shape memory actuators can be improved by increasing the $M_{s}$ temperature up to $60-80^{\circ} \mathrm{C}$ and/or by decreasing their cross section.

\section{NOMENCLATURE}

$A_{s}=$ start temperature for $M \rightarrow A$ transformation $\left({ }^{\circ} \mathrm{C}\right)$

$A_{f}=$ finish temperature for $M \rightarrow A$ transformation $\left({ }^{\circ} \mathrm{C}\right)$

$c_{p}=\mathrm{Ni}-\mathrm{Ti}$ specific heat capacity in absence of transformation $\left(\mathrm{J} / \mathrm{kg} /{ }^{\circ} \mathrm{C}\right)$

$F=$ actuator free surface $\left(\mathrm{m}^{2}\right)$

$G=$ dimensionless hysteresis of transformation

$H=$ dimensionless integral latent heat of transformation

$\Delta H=$ integral latent heat of transformation $(\mathrm{J} / \mathrm{kg})$

$h=$ coefficient of heat exchange between an actuator and surroundings $\left(\mathrm{m}^{2} / \mathrm{K}\right)$

$M_{s}=$ start temperature for the $A \rightarrow M$ transformation $\left({ }^{\circ} \mathrm{C}\right)$

$M_{f}=$ finish temperature for the $A \rightarrow M$ transformation $\left({ }^{\circ} \mathrm{C}\right)$

$n=$ volume fracture of martensite 
$P=$ dimensionless electric power supplied to an actuator

$S=$ dimensionless parameter indicating the difference between transformation and room temperatures

SMA $=$ shape memory alloys

$T=$ temperature of an actuator $\left({ }^{\circ} \mathrm{C}\right)$

$T_{0}=$ surroundings temperature $\left({ }^{\circ} \mathrm{C}\right)$

$T^{\prime}=$ dimensionless temperature of an actuator

$t=$ time (s)

$t_{H}=$ heating time, i.e., the time required for heating of an actuator from $T_{0}$ to $A_{f}(\mathrm{~s})$

$t_{C}=$ cooling time, i.e., the time required for cooling of an actuator from $A_{f}$ to the temperature at which $90 \%$ of the induced deflection (or load) is reset (s)

$t^{\prime}=$ dimensionless time

$t_{0}^{\prime}=$ dimensionless time at which $T^{\prime}$ reaches 0 on cooling

$W=$ electrical power supplied to actuator $(\mathrm{W})$

$\Delta T=$ thermal hysteresis of transformation

$\rho=$ density of $\mathrm{Ni}-\mathrm{Ti}\left(\mathrm{kg} / \mathrm{m}^{3}\right)$

\section{ACKNOWLEDGEMENTS}

The stay of authors in Germany was supported by Alexander von Humboldt-Stiftung (PLP) and Deutscher Akademischer Austauschdienst (EPDS). The authors would like to thank Dr. A. V. Shelyakov for providing ribbons and consultations. The authors acknowledge discussions with Prof. I. Müller and other members of the Thermodynamik team in Technische Universität Berlin.

\section{REFERENCES}

Baehr, H. D. and Stephan, K., 1996. "Wärme—und Stoffübertragung," Springer-Verlag, Berlin.

Beitz, W. and Küttner K. H. 1995. "Dubbel Tashenbuch für den maschinenbau," Springer-Verlag, Berlin.

Bekker, A. and Brinson L. C., 1997. "Temperature-induced phase transformation in a shape memory alloy: phase diagram based kinetics approach," J. Mech. Solids, 45, pp. 949-988.

Bekker, A., Brinson L. C. and Issen K., 1998. "Localized and diffuse thermoinduced phase transformation in 1-D shape memory alloys," J. of Intell. Mat. Syst. \& Struct., 9, pp. 355-365.

Bo, Z., Lagoudas, D. C. and Miller, D., 1999. "Material characterization of SMA actuators under nonproportional thermomechanical loading," J. of Eng. Mat. \& Tech., 121, pp. 75-85.
Boud, J. G. and Lagoudas, D. C., 1996. "A thermodynamic constitutive model for the shape memory materials. Part I: The monolithic shape memory alloys," Int. J. of Plasticity, 12, pp. 805-842.

Brailovski, V., Trochu, F. and Daigneault, G., 1996. "Temporal characteristics of shape memory linear actuators and application to circuit breakers," Mat. \& Design, 17, pp. 151-158.

Brinson, L. C., 1993. "One dimensional constitutive behavior of shape memory alloys," J. of Intell. Mat. Syst. \& Struct., 4(2), pp. 229-249.

Brinson, L. C., Bekker, A. and Hwang, S., 1996. "Deformation of shape memory alloys due to thermo-induced transformation," J. of Intell. Mat. Syst. \& Struct., 7, pp. 97-107.

Lagoudas, D. C. and Bo, Z., 1999. "Thermomechanical modeling of polycrystalline SMAs under cyclic loading, Part II: Material characterization and experimental results for a specific transformation cycle," Int. J. of Eng. Sci., 37, pp. 1141-1174.

Leo, P. H., Shield, T. W. and Bruno, O. P., 1993. "Transient heat transfer effects on the pseudoelastic behavior of shape-memory wires," Acta Metall. Mater., 41, pp. 2477-2485.

Liang, C. and Roger, C. A., 1990. "One-dimensional thermomechanical constitutive relations for shape memory materials," J. of Intell. Mat. Syst. \& Struct., 1(2), pp. 207-234.

Liang, C. and Roger, C. A. 1997. "Design of shape memory alloys," J. of Intell. Mat. Syst. \& Struct., 8, pp. 303-313.

Likhachev, V. A., 1994. "Theory of functional and mechanical properties of materials undergoing reversible martensitic tranformations," Physics of Metals \& Metallography, 77, pp. 120-133.

Miyazaki, S. 1999. "Ni-Ti SMA thin films and their applications," 1st European Conf. on Shape Memory and Superelastic Technologies, Antwerp, Belgium, in press.

Polidori, G., Lachi, M. and Pader, J., 1998. "Unsteady convective heat transfer on a semi-infinite flat surface impulsively heated," Int. Comm. Heat Mass Transfer, 25, pp. 33-42.

Seelecke, S. and Müller, I., 1998. "Thermodynamic aspects of shape memory alloys" in Shape memory alloys-from microstructure to macroscopic properties, Airoldi, G. (ed.), Trans. Tech. Publ., Zürich.

Shaw, J. A. and Kuriakides, S., 1995. "Thermomechanical aspects of NiTi," J. Mech. Phys. Solids, 43, pp. 1243-1281.

Shelyakov, A. V., Antonov, V. A., Bukovsky, Yu. A. and Matveeva, N. M., 1994. "Optical devices based on shape memory effect for signal processing," Proc. 1st Int. Conf. on Shape Memory and Superelastic Technologies, Pacific Grove, California, USA, pp. 335-340.

Tanaka, Y. and Nagaki, S., 1982. "A thermomechanical description of materials with internal variables in the process of phase transitions," Ingenier Archiv., 51, pp. 287-299.

Wirtz, R. A., Gordaninejad, F. and Wu, W., 1995. "Free response of a thermally driven, composite actuator," J. Intell. Mat. Systems and Structures, 5, pp. 364-371.

Yaeger, J. R., 1990. "Electrical actuators: alloy selection, processing and evaluation" in "Engineering Aspects of Shape Memory Alloys," ed. T. W. Duerig et al., Butterworth-Heinemann, London, pp. 219-233. 